PROCEEDINGS OF THE

AMERICAN MATHEMATICAL SOCIETY

Volume 139, Number 12, December 2011, Pages 4173-4179

S 0002-9939(2011)10837-2

Article electronically published on April 5, 2011

\title{
THE BRAUER GROUP OF MODULI SPACES OF VECTOR BUNDLES OVER A REAL CURVE
}

\author{
INDRANIL BISWAS, NORBERT HOFFMANN, AMIT HOGADI, \\ AND ALEXANDER H. W. SCHMITT \\ (Communicated by Lev Borisov)
}

\begin{abstract}
Let $X$ be a geometrically connected smooth projective curve of genus $g_{X} \geq 2$ over $\mathbb{R}$. Let $M(r, \xi)$ be the coarse moduli space of geometrically stable vector bundles $E$ over $X$ of rank $r$ and determinant $\xi$, where $\xi$ is a real point of the Picard variety $\operatorname{Pic}^{d}(X)$. If $g_{X}=r=2$, then let $d$ be odd. We compute the Brauer group of $M(r, \xi)$.
\end{abstract}

\section{INTRODUCTION}

Let $X_{\mathbb{C}}$ be a connected smooth projective curve of genus $g_{X} \geq 2$ over $\mathbb{C}$. Fix integers $r \geq 2$ and $d$. Given a line bundle $\xi_{\mathbb{C}}$ of degree $d$ over $X_{\mathbb{C}}$, we denote by $M\left(r, \xi_{\mathbb{C}}\right)$ the coarse moduli space of stable vector bundles over $X_{\mathbb{C}}$ of rank $r$ and determinant $\xi_{\mathbb{C}}$.

The Picard group of such moduli spaces has been studied intensively; see for example [DN, $\mathrm{KN}, \mathrm{LS}$, , So, $\mathrm{BLS}, \mathrm{Fa}, \mathrm{Te}, \mathrm{BHo1}$. We view the Brauer group as a natural higher order analogue of the Picard group. It is related to the classical rationality problem $\mathrm{CS}$.

We assume that $d$ is odd if $g_{X}=r=2$; otherwise $d$ is arbitrary. The Brauer group of $M\left(r, \xi_{\mathbb{C}}\right)$ has been computed in $\mathrm{BBGN}$; the result is a canonical isomorphism

$$
\operatorname{Br}\left(M\left(r, \xi_{\mathbb{C}}\right)\right) \cong \mathbb{Z} / \operatorname{gcd}(r, d)
$$

The corresponding generator $\beta_{\mathbb{C}} \in \operatorname{Br}\left(M\left(r, \xi_{\mathbb{C}}\right)\right)$ can be viewed as the obstruction against the existence of a Poincaré bundle, or universal vector bundle, over $M\left(r, \xi_{\mathbb{C}}\right) \times X_{\mathbb{C}}$.

Now suppose $X_{\mathbb{C}}=X \otimes_{\mathbb{R}} \mathbb{C}$ for a smooth projective curve $X$ over $\mathbb{R}$. Then some of the above moduli spaces carry interesting real algebraic structures, and there has been a growing interest in understanding these structures $[\mathrm{BhB}, \mathrm{BHH}, \mathrm{BHu}, \mathrm{Sch}$. In this paper we compute the Brauer group of such real algebraic moduli spaces.

More precisely, assume that the line bundle $\xi_{\mathbb{C}}$ comes from a real point $\xi$ of the Picard variety $\operatorname{Pic}^{d}(X)$. Let $M(r, \xi)$ be the coarse moduli space of geometrically stable vector bundles $E$ over $X$ of rank $r$ and determinant $\xi$. It is a smooth quasiprojective variety over $\mathbb{R}$, with $M(r, \xi) \otimes_{\mathbb{R}} \mathbb{C} \cong M\left(r, \xi_{\mathbb{C}}\right)$; see Section 2 . Our main result, Theorem 3.3, describes the Brauer group of $M(r, \xi)$ as follows.

Received by the editors August 19, 2010 and, in revised form, October 13, 2010.

2010 Mathematics Subject Classification. Primary 14F22, 14D20, 14P99.

Key words and phrases. Brauer group, moduli space, real algebraic curve.

(C)2011 American Mathematical Society Reverts to public domain 28 years from publication 4173 
Theorem 1.1. With $\chi:=r\left(1-g_{X}\right)+d$, there is a canonical isomorphism

$\operatorname{Br}(M(r, \xi)) \cong \begin{cases}\mathbb{Z} / \operatorname{gcd}(r, \chi) \oplus \mathbb{Z} / 2 & \text { if } \xi \text { comes from a line bundle defined over } \mathbb{R}, \\ \mathbb{Z} / \operatorname{gcd}(2 r, \chi) & \text { otherwise. }\end{cases}$

Note that $\operatorname{gcd}(r, \chi)=\operatorname{gcd}(r, d)$. The $\operatorname{groups} \mathbb{Z} / \operatorname{gcd}(r, \chi)$ and $\mathbb{Z} / \operatorname{gcd}(2 r, \chi)$ are generated by a canonical class $\beta \in \operatorname{Br}(M(r, \xi))$, the obstruction against a Poincaré bundle over $M(r, \xi) \times X$. The order of this obstruction class $\beta$ is computed in Proposition 3.2. The remaining direct summand $\mathbb{Z} / 2$ comes from the Brauer group of $\mathbb{R}$.

\section{Moduli of Vector Bundles over a ReAl CuRve}

Let $X$ be a geometrically connected smooth projective algebraic curve of genus $g_{X} \geq 2$ defined over $\mathbb{R}$. We will denote the base change from $\mathbb{R}$ to $\mathbb{C}$ by a subscript $\mathbb{C}$. In particular, $X_{\mathbb{C}}:=X \otimes_{\mathbb{R}} \mathbb{C}$ is the associated algebraic curve over $\mathbb{C}$.

Let $\sigma: \mathbb{C} \longrightarrow \mathbb{C}$ denote complex conjugation. The involutive morphism of schemes

$$
\sigma_{X}:=\operatorname{id}_{X} \otimes \sigma: X_{\mathbb{C}} \longrightarrow X_{\mathbb{C}}
$$

lies over $\sigma: \mathbb{C} \longrightarrow \mathbb{C}$. The closed points of $X_{\mathbb{C}}$ fixed by $\sigma_{X}$ are the real points of $X$.

Let $\xi$ be a real point of the Picard variety $\underline{\operatorname{Pic}}(X)$. Viewing the associated complex point $\xi_{\mathbb{C}}$ of $\underline{\operatorname{Pic}}\left(X_{\mathbb{C}}\right)$ as a line bundle over $X_{\mathbb{C}}$, we have $\xi_{\mathbb{C}} \cong \sigma_{X}^{*}\left(\xi_{\mathbb{C}}\right)$.

A real (respectively, quaternionic) structure on $\xi_{\mathbb{C}}$ is by definition an isomorphism

$$
\eta: \xi_{\mathbb{C}} \longrightarrow \sigma_{X}^{*}\left(\xi_{\mathbb{C}}\right)
$$

of line bundles over $X_{\mathbb{C}}$ with $\sigma_{X}^{*} \eta \circ \eta=\mathrm{id}_{\xi_{\mathbb{C}}}$ (respectively, $\sigma_{X}^{*} \eta \circ \eta=-\mathrm{id}_{\xi_{\mathbb{C}}}$ ). The line bundle $\xi_{\mathbb{C}}$ admits either a real structure $\eta$ or a quaternionic structure $\eta$, and in both cases the resulting pair $\left(\xi_{\mathbb{C}}, \eta\right)$ is uniquely determined up to an isomorphism; cf. for example [Ve, Proposition 2.5] or [BHH, Proposition 3.1].

The real point $\xi$ of $\underline{\operatorname{Pic}}(X)$ is called quaternionic if $\xi_{\mathbb{C}}$ admits a quaternionic structure. Otherwise, $\overline{\xi_{\mathbb{C}}}$ admits a real structure, so we can view $\xi$ as a real line bundle over $X$.

A vector bundle $E$ over $X$ is called geometrically stable if the vector bundle $E_{\mathbb{C}}$ over $X_{\mathbb{C}}$ is stable. Not every stable vector bundle $E$ over $X$ is geometrically stable, but it is always geometrically polystable. Fix integers $r \geq 2$ and $d$. We denote by

$$
\mathcal{M}(r, d) \supset \mathcal{M}(r, d)^{s} \longrightarrow M(r, d)
$$

the moduli stack of vector bundles $E$ over $X$ of rank $r$ and degree $d$, the open substack of geometrically stable $E$, and their coarse moduli scheme, respectively. Since geometrically stable $E$ have only scalar automorphisms, $\mathcal{M}(r, d)^{s}$ is a gerbe with band $\mathbb{G}_{\mathrm{m}}$ over $M(r, d)$.

Let $\mathcal{L}($ det $)$ denote the determinant of the cohomology line bundle over $\mathcal{M}(r, d)$. Its fiber over the moduli point of a vector bundle $E$ is by definition $\operatorname{det} \mathrm{H}^{0}(E) \otimes$ $\operatorname{det}^{-1} \mathrm{H}^{1}(E)$.

All three moduli spaces or stacks in (2.1) come with a determinant map to the Picard variety $\underline{\operatorname{Pic}}^{d}(X)$. Given a real point $\xi$ of $\underline{\operatorname{Pic}}^{d}(X)$, we denote by

$$
\mathcal{M}(r, \xi) \supset \mathcal{M}(r, \xi)^{s} \longrightarrow M(r, \xi)
$$


the corresponding fibers over $\xi$. So $M(r, \xi)$ is a smooth quasiprojective variety over $\mathbb{R}$, whose base change $M(r, \xi)_{\mathbb{C}}$ is the moduli space of stable vector bundles over $X_{\mathbb{C}}$ of rank $r$ and determinant $\xi_{\mathbb{C}}$. By restriction, $\mathcal{M}(r, \xi)^{s}$ is a gerbe with band $\mathbb{G}_{\mathrm{m}}$ over $M(r, \xi)$.

Suppose for the moment that $\xi$ is a real line bundle. Then we can define a line bundle $\mathcal{L}(\xi)$ over $\mathcal{M}(r, \xi)$ whose fiber over the moduli point of a vector bundle $E$ is $\operatorname{Hom}(\xi, \operatorname{det} E)$. To state this more precisely, let $S$ be a scheme over $\mathbb{R}$. Then the pullback of $\mathcal{L}(\xi)$ along the classifying morphism $S \longrightarrow \mathcal{M}(r, \xi)$ of a vector bundle $\mathcal{E}$ over $X \times S$ is by definition the line bundle $\operatorname{pr}_{2, *}\left(\operatorname{pr}_{1}^{*} \xi^{-1} \otimes \operatorname{det} \mathcal{E}\right)$ over $S$. This defines a line bundle $\mathcal{L}(\xi)$ over $\mathcal{M}(r, \xi)$.

Now suppose that $\xi$ is quaternionic. Then the same recipe defines a line bundle over $\mathcal{M}(r, \xi)_{\mathbb{C}}$ endowed with a quaternionic structure. We denote this pair again by $\mathcal{L}(\xi)$.

In both cases, $\mathcal{L}(\xi)$ gives us a line bundle $\mathcal{L}(\xi)_{\mathbb{C}}$ over $\mathcal{M}(r, \xi)_{\mathbb{C}}$. If we trivialize the fiber of $\xi_{\mathbb{C}}$ over one closed point $x_{0} \in X_{\mathbb{C}}$, we can identify $\mathcal{L}(\xi)_{\mathbb{C}}$ with the line bundle whose fiber at the moduli point of a vector bundle $E_{\mathbb{C}}$ over $X_{\mathbb{C}}$ is the fiber of $\operatorname{det} E_{\mathbb{C}}$ over $x_{0}$.

Proposition 2.1. The Picard group $\operatorname{Pic}(\mathcal{M}(r, \xi))$ is generated by

i) $\mathcal{L}(\mathrm{det})$ and $\mathcal{L}(\xi)$ if $\xi$ is a real line bundle,

ii) $\mathcal{L}(\mathrm{det})$ and $\mathcal{L}(\xi)^{\otimes 2}$ if $\xi$ is quaternionic.

The restrictions of these line bundles also generate $\operatorname{Pic}\left(\mathcal{M}(r, \xi)^{s}\right)$.

Proof. Let $\widetilde{\mathcal{M}}\left(r, \xi_{\mathbb{C}}\right)$ denote the moduli stack of vector bundles $E$ of rank $r$ over $X_{\mathbb{C}}$ together with an isomorphism $\xi_{\mathbb{C}} \cong \operatorname{det} E$. The forgetful map

$$
\pi: \widetilde{\mathcal{M}}\left(r, \xi_{\mathbb{C}}\right) \longrightarrow \mathcal{M}(r, \xi)_{\mathbb{C}}
$$

is the $\mathbb{G}_{\mathrm{m}}$-torsor given by the line bundle $\mathcal{L}(\xi)_{\mathbb{C}}$. It is easy to check that the kernel of

$$
\pi^{*}: \operatorname{Pic}\left(\mathcal{M}(r, \xi)_{\mathbb{C}}\right) \longrightarrow \operatorname{Pic}\left(\widetilde{\mathcal{M}}\left(r, \xi_{\mathbb{C}}\right)\right)
$$

is generated by $\mathcal{L}(\xi)_{\mathbb{C}}$; cf. the proof of [BL, Lemma 7.8]. The Picard group of $\widetilde{\mathcal{M}}\left(r, \xi_{\mathbb{C}}\right)$ is generated by $\pi^{*}\left(\mathcal{L}(\text { det })_{\mathbb{C}}\right)$, according to $\mathrm{BL}$, Remark 7.11 and Proposition 9.2].

This shows that $\operatorname{Pic}\left(\mathcal{M}(r, \xi)_{\mathbb{C}}\right)$ is generated by $\mathcal{L}(\text { det })_{\mathbb{C}}$ and $\mathcal{L}(\xi)_{\mathbb{C}}$. We have just seen that all these line bundles admit a real or quaternionic structure. This real or quaternionic structure is unique, since $\Gamma\left(\mathcal{M}(r, \xi)_{\mathbb{C}}, \mathcal{O}^{*}\right)=\mathbb{C}^{*}$. It follows that $\operatorname{Pic}(\mathcal{M}(r, \xi))$ is the subgroup of line bundles in $\operatorname{Pic}\left(\mathcal{M}(r, \xi)_{\mathbb{C}}\right)$ which are real, not quaternionic. Hence $\operatorname{Pic}(\mathcal{M}(r, \xi))$ is generated by the line bundles as claimed.

As $\mathcal{M}(r, \xi)$ is smooth, the restriction map $\operatorname{Pic}(\mathcal{M}(r, \xi)) \longrightarrow \operatorname{Pic}\left(\mathcal{M}(r, \xi)^{s}\right)$ is surjective; cf. for example [BHo2, Lemma 7.3]. So these line bundles also generate $\operatorname{Pic}\left(\mathcal{M}(r, \xi)^{s}\right)$.

Now let $\mathcal{M} \longrightarrow M$ be a gerbe with band $\mathbb{G}_{\mathrm{m}}$ over an irreducible Noetherian scheme $M$. As a basic example, we have the gerbe $\mathcal{M}(r, d)^{s} \longrightarrow M(r, d)$ in mind.

Definition 2.2. Let $\mathcal{L}$ be a line bundle over $\mathcal{M}$. Then the automorphism groups $\mathbb{G}_{\mathrm{m}}$ in $\mathcal{M}$ act on the fibers of $\mathcal{L}$. These $\mathbb{G}_{\mathrm{m}}$ act by the same power $w \in \mathbb{Z}$ on every fiber of $\mathcal{L}$, since $\mathcal{M}$ is connected. The integer $w$ is called the weight of $\mathcal{L}$. 
The weight of a quaternionic line bundle $\mathcal{L}$ is by definition the weight of the associated complex line bundle $\mathcal{L}_{\mathbb{C}}$. For example, the real or quaternionic line bundle $\mathcal{L}(\xi)$ over $\mathcal{M}(r, \xi)^{s}$ has weight $r$. The real line bundle $\mathcal{L}($ det $)$ over $\mathcal{M}(r, d)^{s}$ has weight

$$
\chi:=r\left(1-g_{X}\right)+d
$$

according to Riemann-Roch. Consider the integers

$$
\chi^{\prime}:=\chi / \operatorname{gcd}(r, \chi) \quad \text { and } \quad r^{\prime}:=r / \operatorname{gcd}(r, \chi) .
$$

The real or quaternionic line bundle

$$
\mathcal{L}(\Theta):=\mathcal{L}(\operatorname{det})^{\otimes-r^{\prime}} \otimes \mathcal{L}(\xi)^{\otimes \chi^{\prime}}
$$

over $\mathcal{M}(r, \xi)^{s}$ has weight 0 . Hence it descends to a real or quaternionic line bundle over $M(r, \xi)$, which we again denote by $\mathcal{L}(\Theta)$. The line bundle $\mathcal{L}(\Theta)_{\mathbb{C}}$ is ample on $M(r, \xi)_{\mathbb{C}}$, and it generates the Picard group $\operatorname{Pic}\left(M(r, \xi)_{\mathbb{C}}\right)$ according to $[\mathrm{DN}$, Théorèmes A \& B].

Proposition 2.3. The Picard group $\operatorname{Pic}(M(r, \xi))$ is generated by

i) $\mathcal{L}(\Theta)$ if $\xi$ is a real line bundle or $\chi^{\prime}$ is even,

ii) $\mathcal{L}(\Theta)^{\otimes 2}$ if $\xi$ is quaternionic and $\chi^{\prime}$ is odd.

Proof. The line bundles over $M(r, \xi)$ are the line bundles of weight 0 over $\mathcal{M}(r, \xi)^{s}$. According to Proposition 2.1, these are of the form $\mathcal{L}(\text { det })^{\otimes a} \otimes \mathcal{L}(\xi)^{\otimes b}$ with $a \chi+b r=$ 0 , where moreover $b$ has to be even if $\xi$ is quaternionic.

\section{The Brauer group}

The Brauer group $\operatorname{Br}(S)$ of a Noetherian scheme $S$ is by definition the abelian group of Azumaya algebras over $S$ up to Morita equivalence. It is a torsion group, and it embeds canonically into the étale cohomology group $\mathrm{H}_{\text {ét }}^{2}\left(S, \mathbb{G}_{\mathrm{m}}\right)$.

If $S$ is smooth and quasiprojective over a field, then $\mathrm{H}_{\text {ét }}^{2}\left(S, \mathbb{G}_{\mathrm{m}}\right)$ is also a torsion group $\left[\mathrm{Gr}\right.$, Proposition 1.4], and the embedding of $\operatorname{Br}(S)$ into $\mathrm{H}_{\text {ét }}^{2}\left(S, \mathbb{G}_{\mathrm{m}}\right)$ is an isomorphism d.J.

Our aim is to compute the Brauer group of the real moduli space $M(r, \xi)$. Let

$$
\beta \in \mathrm{H}_{\text {ét }}^{2}\left(M(r, \xi), \mathbb{G}_{\mathrm{m}}\right)=\operatorname{Br}(M(r, \xi))
$$

denote the class given by the gerbe $\mathcal{M}(r, \xi)^{s} \longrightarrow M(r, \xi)$ with band $\mathbb{G}_{\mathrm{m}}$. Since a section of this gerbe would yield a Poincaré bundle over $M(r, \xi) \times X$, we can view the class $\beta$ as the obstruction against the existence of such a Poincaré bundle.

Remark 3.1. Choose an effective divisor $D \subset X$ defined over $\mathbb{R}$, for example a closed point in $X$. The Brauer class $\beta$ over $M(r, \xi)$ can also be described by the Azumaya algebra with fibers $\operatorname{End~}^{0}\left(D,\left.E\right|_{D}\right)$, or by the projective bundle with fibers $\mathbb{P} \mathrm{H}^{0}\left(D,\left.E\right|_{D}\right)$.

We first compute the exponent of $\beta$, i.e., the order of $\beta$ as an element in the torsion group $\operatorname{Br}(M(r, \xi))$. This will in particular re-prove results of [BHu, Section 5].

Proposition 3.2. Let $\xi$ be a real point of the Picard variety $\underline{\mathrm{Pic}}^{d}(X)$.

i) If $\xi$ is a real line bundle, then $\beta \in \operatorname{Br}(M(r, \xi))$ has exponent $\operatorname{gcd}(r, \chi)$.

ii) If $\xi$ is quaternionic, then $\beta \in \operatorname{Br}(M(r, \xi))$ has exponent $\operatorname{gcd}(2 r, \chi)$. 
Proof. An integer $n \in \mathbb{Z}$ annihilates the class $\beta \in \mathrm{H}_{\text {ét }}^{2}\left(M(r, \xi), \mathbb{G}_{\mathrm{m}}\right)$ of the gerbe $\mathcal{M}(r, \xi)^{s}$ if and only if there is a line bundle $\mathcal{L}$ over $\mathcal{M}(r, \xi)^{s}$ which has weight $n$; see for example [Ho, Lemma 4.9]. Hence the claim follows from Proposition 2.1. Let

We denote by $\mathbb{Z} \cdot \beta \subseteq \operatorname{Br}(M(r, \xi))$ the subgroup generated by the class $\beta$ in (3.1).

$$
f: M(r, \xi) \longrightarrow \operatorname{Spec}(\mathbb{R})
$$

be the structure morphism. Recall that $\operatorname{Br}(\mathbb{R}) \cong \mathbb{Z} / 2$, the nontrivial element being the class $[\mathbb{H}] \in \operatorname{Br}(\mathbb{R})$ of the quaternion algebra $\mathbb{H}=\mathbb{R} \oplus \mathbb{R} \cdot i \oplus \mathbb{R} \cdot j \oplus \mathbb{R} \cdot k$.

Theorem 3.3. Let $\xi$ be a real point of $\underline{\operatorname{Pic}}^{d}(X)$, with $d$ odd if $g_{X}=r=2$. We have

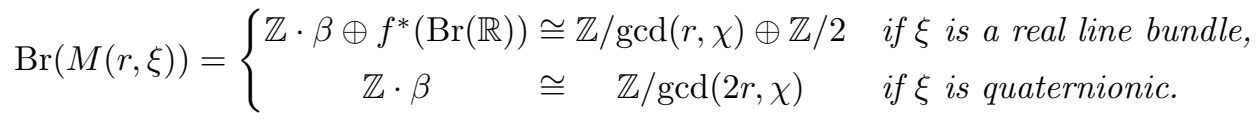

Proof. The structure morphism $f$ in (3.2) yields a Leray spectral sequence

$$
E_{2}^{p, q}=\mathrm{H}_{\text {êt }}^{p}\left(\mathbb{R}, \mathrm{R}^{q} f_{*} \mathbb{G}_{\mathrm{m}}\right) \Rightarrow \mathrm{H}_{\text {ét }}^{p+q}\left(M(r, \xi), \mathbb{G}_{\mathrm{m}}\right) .
$$

We have $\mathrm{R}^{1} f_{*} \mathbb{G}_{\mathrm{m}}=\operatorname{Pic}\left(M(r, \xi)_{\mathbb{C}}\right) \cong \mathbb{Z}$. The action of $\operatorname{Gal}(\mathbb{C} / \mathbb{R})=\mathbb{Z} / 2$ on it is trivial, for example because it preserves ampleness. From this we deduce

$$
E_{2}^{1,1}=\mathrm{H}_{\text {ét }}^{1}(\mathbb{R}, \mathbb{Z})=\operatorname{Hom}(\mathbb{Z} / 2, \mathbb{Z})=0 .
$$

Hence the spectral sequence (3.3) provides in particular an exact sequence

$$
\mathrm{H}_{\text {ét }}^{1}\left(M(r, \xi), \mathbb{G}_{\mathrm{m}}\right) \longrightarrow E_{2}^{0,1} \longrightarrow E_{2}^{2,0} \longrightarrow \mathrm{H}_{\text {ét }}^{2}\left(M(r, \xi), \mathbb{G}_{\mathrm{m}}\right) \longrightarrow E_{2}^{0,2} .
$$

Using $f_{*} \mathbb{G}_{\mathrm{m}}=\mathbb{G}_{\mathrm{m}}$ and $\mathrm{R}^{2} f_{*} \mathbb{G}_{\mathrm{m}}=\operatorname{Br}\left(M(r, \xi)_{\mathbb{C}}\right)$, we thus obtain an exact sequence

$$
\operatorname{Pic}(M(r, \xi)) \stackrel{g^{1}}{\longrightarrow} \operatorname{Pic}\left(M(r, \xi)_{\mathbb{C}}\right) \longrightarrow \operatorname{Br}(\mathbb{R}) \stackrel{f^{*}}{\longrightarrow} \operatorname{Br}(M(r, \xi)) \stackrel{g^{2}}{\longrightarrow} \operatorname{Br}\left(M(r, \xi)_{\mathbb{C}}\right),
$$

where $g^{1}$ and $g^{2}$ are pullback maps along the projection $g: M(r, \xi)_{\mathbb{C}} \longrightarrow M(r, \xi)$. Note that $g^{2}$ is surjective, since $g^{2}(\beta)=\beta_{\mathbb{C}}$ generates $\operatorname{Br}\left(M(r, \xi)_{\mathbb{C}}\right)$ by [BBGN].

Suppose that $\xi$ is a real line bundle. Then $g^{1}$ is surjective due to Proposition 2.3. so $f^{*}$ is injective. Since $\beta$ has the same exponent as its image $\beta_{\mathbb{C}}$ by Proposition 3.2 , it follows that $\operatorname{Br}(M(r, \xi))$ is the direct sum of its subgroups $\mathbb{Z} \cdot \beta$ and $f^{*}(\operatorname{Br}(\mathbb{R}))$, as required.

Now suppose that $\xi$ is quaternionic and that $\chi^{\prime}=\chi / \operatorname{gcd}(r, \chi)$ is even. Then $f^{*}$ is injective as before, but the exponent $\operatorname{gcd}(2 r, \chi)$ of $\beta$ is twice the exponent $\operatorname{gcd}(r, \chi)$ of its image $\beta_{\mathbb{C}}$. Hence $\operatorname{gcd}(r, \chi) \cdot \beta=f^{*}([\mathbb{H}])$, and the class $\beta$ generates $\operatorname{Br}(M(r, \xi))$.

Finally, suppose that $\xi$ is quaternionic and that $\chi^{\prime}$ is odd. Then the cokernel of $g^{1}$ has two elements according to Proposition 2.3, so $f^{*}$ is the zero map, and $g^{2}$ is an isomorphism. In particular, the class $\beta$ again generates $\operatorname{Br}(M(r, \xi))$. 


\section{ACKNOWLEDGEMENTS}

Part of this work was done while the first author was visiting Freie Universität Berlin, with support from the SFB 647: Space - Time - Matter. The second and the fourth authors were also supported by SFB 647 .

\section{REFERENCES}

[BBGN] V. Balaji, I. Biswas, O. Gabber and D. S. Nagaraj, Brauer obstruction for a universal vector bundle, Comp. Rend. Math. Acad. Sci. Paris 345 (2007), 265-268. MR 2353678 (2008h:14031)

[BL] A. Beauville and Y. Laszlo, Conformal blocks and generalized theta functions. Commun. Math. Phys. 164 (1994), 385-419. MR:1289330(95k:14011)

[BLS] A. Beauville, Y. Laszlo and C. Sorger, The Picard group of the moduli of $G$-bundles on a curve, Compositio Math. 112 (1998), 183-216. MR.1626025(99i:14011)

[BhB] U. N. Bhosle and I. Biswas, Stable real algebraic vector bundles over a Klein bottle. Trans. Amer. Math. Soc. 360 (2008), 4569-4595. MR2403697 (2009f:14063)

[BHo1] I. Biswas and N. Hoffmann, The line bundles on moduli stacks of principal bundles on a curve, Documenta Math. 15 (2010), 35-72. MR2628848

[BHo2] I. Biswas and N. Hoffmann, Poincaré families of $G$-bundles on a curve, preprint arXiv:1001.2123, available at www.arXiv.org, to appear in Math. Ann.

[BHH] I. Biswas, J. Huisman and J. Hurtubise, The moduli space of stable vector bundles over a real algebraic curve, Math. Ann. 347 (2010), 201-233. MR2593289

$[\mathrm{BHu}]$ I. Biswas and J. Hurtubise, Universal vector bundle over the reals, preprint arXiv: 0909.0041, available at www.arXiv.org, to appear in Trans. Amer. Math. Soc.

[CS] J.-L. Colliot-Thélène and J.-J. Sansuc, The rationality problem for fields of invariants under linear algebraic groups (with special regard to the Brauer group), in: V. Mehta, editor, Algebraic groups and homogeneous spaces (Mumbai, 2004), pages 113-186, 2007. MR2348904 (2008m:13010)

[dJ] A. de Jong: A result of Gabber, preprint, http://www.math.columbia.edu/ dejong/

[DN] J.-M. Drezet and M. S. Narasimhan, Groupe de Picard des variétés de modules de fibrés semi-stables sur les courbes algébriques, Invent. Math. 97 (1989), 53-94. MR999313 (90d:14008)

[Fa] G. Faltings, Algebraic loop groups and moduli spaces of bundles, J. Eur. Math. Soc. (JEMS) 5 (2003), 41-68. MR1961134 (2003k:14011)

[Gr] A. Grothendieck, Le groupe de Brauer II: Théorie cohomologique, Dix Exposés sur la Cohomologie des Schémas, 67-87. North-Holland, Amsterdam, 1968. MR0244270 (39:5586b)

[Ho] N. Hoffmann, Rationality and Poincaré families for vector bundles with extra structure on a curve, Int. Math. Res. Not., Article ID rnm010 (2007), 30 pp. MR 2337034 (2009b:14066)

[KN] S. Kumar and M. S. Narasimhan, Picard group of the moduli spaces of $G$-bundles, Math. Ann. 308 (1997), 155-173. MR1446205 (98d:14014)

[LS] Y. Laszlo and C. Sorger, The line bundles on the moduli of parabolic G-bundles over curves and their sections, Ann. Sci. École Norm. Sup. (4) 30 (1997), 499-525. MR 1456243 (98f:14007)

[Sch] F. Schaffhauser, Moduli varieties of real and quaternionic vector bundles over a curve, preprint arXiv:1003.5285, available at www.arXiv.org

[So] C. Sorger, On moduli of $G$-bundles of a curve for exceptional G, Ann. Sci. École Norm. Sup. (4) 32 (1999), 127-133. MR1670528 (99m:14031)

$[\mathrm{Te}]$ C. Teleman, Borel-Weil-Bott theory on the moduli stack of $G$-bundles over a curve, Invent. Math. 134 (1998), 1-57. MR1646586 (2000b:14014)

[Ve] J.-L. Verdier, Instantons. Les équations de Yang-Mills, Séminaire École Norm. Sup. 1977-1978, 105-134. Astérisque 71-72, Société Mathématique de France, Paris, 1980. MR:589891 (84j:14026) 
School of Mathematics, Tata Institute of Fundamental Research, Homi Bhabha ROAD, Bombay 400005, India

E-mail address: indranil@math.tifr.res.in

Institut für Mathematik, Freie Universität, Arnimallee 3, 14195 Berlin, Germany

E-mail address: norbert.hoffmann@fu-berlin.de

School of Mathematics, Tata Institute of Fundamental Research, Homi Bhabha RoAD, Bombay 400005, INDia

E-mail address: amit@math.tifr.res.in

Institut für Mathematik, Freie Universität, Arnimallee 3, 14195 Berlin, Germany

E-mail address: alexander.schmitt@fu-berlin.de 